\title{
Review of: "Evaluating Machine Learning Classifiers for Glaucoma Referral Decision Support in Primary Care Settings"
}

Jothi Prabha Appadurai

Potential competing interests: The author(s) declared that no potential competing interests exist.

Recommendation: Minor Revisions required:

Comments:

In the current study, the authors have used machine learning classifiers(Support vector machine, logistic regression, and adaptive boosting) to predict self-reported glaucoma. The goal was to explore the potential for combining readily accessible patient data with simple IOP measurement in a non-eye care setting to inform referral decisions and, thus, increase the number of glaucoma suspects evaluated early by an ophthalmologist.

The manuscript is well-organized and the idea seems nice. As presented by the results, the features which contribute most to the F1 score are IOP and age. The topic is interesting however, there are minor points for revisions.

Some comments and suggestions are as follows:

1. The author should emphasize on the advantages of using this set of features predict self-reported glaucoma

2. Is hyperparameter optimization done for the ensemble method Adaboost,? If so the details of hyperparameter settings in the framework should be mentioned

3. The authors should justify why they have chosen Support Vector Machine, Logistic Regression and Adaboost

4. Some references from recent year research can be added.

5. The paper is generally well written. However, at places there are presentation and grammar issues. Authors should carefully read the paper before submitting the final version. 